\title{
Stadtteilbegehung mit älteren Menschen
}

\section{Ein Modell zur Partizipation im Alter}

\section{LISA BEHRET}

Lisa Behret ist Absolventin der Dualen Hochschule Baden-Württemberg in Villingen-Schwenningen im Bachelor Soziale Arbeit (2011) und derzeit Masterstudentin für Soziale Arbeit an der Evangelischen Hochschule Dresden.

lisa_behret@gmx.de

\author{
Bei der Gestaltung von Stadtteilen und anderen \\ Sozialräumen stellt sich die Frage nach praktischen \\ Methoden bei der partizipatorischen Beteiligung \\ insbesondere von älteren Menschen. Mit dem \\ Angebot einer entsprechend vorbereiteten, \\ durchgeführten und dokumentierten Stadtteilbegehung \\ kann diese Personengruppe aktiviert und als \\ "Mitforschende" an Planungen beteiligt werden.
}

Das Alter ist mehr als eine Anzahl an erreichten Lebensjahren. Es schließt individuelle soziale und kulturelle Aspekte ein. Das Alter ist eine Differenzkategorie und steht immer in einem Verhältnis: Alt ist jemand in Relation oder im Gegensatz zu jung.

Die Bertelsmann Stiftung definiert: »Das Alter ist primär eine soziale Kategorie, in der sich die für eine gegebene Gesellschaft zu einem bestimmten Zeitpunkt charakteristische Zuordnung von sozialen Rollen zu den verschiedenen Lebensaltern sowie zeitgeschichtliche Ereignisse und Entwicklungen widerspiegeln.«(1)

Die Lebensphase Alter ist geprägt von gesellschaftlichen Vorstellungen des Zustandes Altsein, vom Prozess des Alterns und von der sozialen Gruppe älterer Menschen. Solche sogenannten Altersbilder sind - ebenso wie Altersgrenzen - soziale Konstruktionen.

(2) Knopp und Deinet nehmen eine Einteilung älterer Menschen in vier Gruppen vor:

- die Gruppe der Älteren, die noch im Berufsleben stehen und sich auf die nachberufliche Phase vorbereiten

- diejenigen, die sich in der nachberuflichen Phase befinden und noch sehr mobil sind

- die Gruppe mit abnehmender oder eingeschränkter Mobilität sowie
- die Hochbetagten, die in unterschiedlichem Maße Unterstützung und Pflege benötigen. (3)

\section{Dimensionen des Alters}

Das Alter ist mehrdimensional und zurzeit von einem Strukturwandel geprägt. Aus dem Doppeleffekt eines früheren Ausstiegs aus dem Berufsleben und einer steigenden Lebenserwartung resultiert eine zeitliche Ausdehnung der Altersphase auf nicht selten 30 Jahre. (4) Hochaltrigkeit ist als herausragendes Merkmal des Strukturwandels des Alters zu sehen. Kennzeichnend für diese Phase jenseits des 80. Lebensjahres ist eine Kumulation von Einschränkungen und Erkrankungen.

Die Binnendifferenzierung der Lebensphase Alter macht sich an typischen Phasen mit charakteristischen Ereignissen fest, wie beispielsweise das Ausscheiden aus dem Erwerbsleben, das Großelterndasein, beginnende funktionale und gesundheitliche Einschränkungen bis hin zur Pflegebedürftigkeit und dem Tod des Partners.

Bezeichnend für den Strukturwandel ist außerdem eine stetig zunehmende Feminisierung des Alters. Naegele zeigt auf, dass der Frauenanteil der 60 Jahre alten und älteren Menschen etwa drei Fünftel ausmacht, der Männeranteil hingegen nur zwei Fünftel in dieser Altersgruppe beträgt. (5) 
In der Bundesrepublik leben circa 40 Prozent der Menschen über 65 Jahren alleine, wobei 85 Prozent dieser SingleHaushalte Frauen sind. (6) Mit zunehmendem Alter ist mit dieser Singularisierung eine Hilfebedürftigkeit und Abhängigkeit von Dritten verbunden.

\section{Partizipation als Forschungs- und Handlungskonzept}

Nach Gintzel impliziert Partizipation eine Vielzahl an Ansätzen der bewussten Beteiligung von Menschen mit dem Ziel von Teilhabe, Mitgestaltung, Mitwirkung und Mitverantwortung. (7) Für Schnurr ist Partizipation im Kontext Sozialer Arbeit die Beteiligung von Adressaten an Entscheidungen über Bedarfe, Angebots- und Leistungsstrukturen sowie über individuelle Prozesse der Leistungserbringung. Im Weiteren geht es um die Ausübung von Wahlfreiheit im Bezug auf Spezifikationen von Leistungen. (8)

Partizipation ist als Strukturmaxime zu sehen, da sie Umfang, Art und Form der Begleitung und Unterstützung von Adressaten bestimmt (9), als auch die Beziehung sowie Interaktion zwischen Adressaten und Sozialarbeitern gestaltet. Im Fokus stehen die Aktivierung der Adressaten und die Motivation zu selbstbestimmtem, reflektiertem Handeln. Partizipation ist in diesem Sinne Strukturvoraussetzung und Erfolgsbedingung Sozialer Arbeit. (10)

Die Diskussion zu Partizipation wird vor allem in der Kinder- und Jugendhilfe geführt. (11) Die folgenden Zielsetzungen von Partizipation sind dort entlehnt und auf die Altenarbeit übertragen: Eröffnung von Chancen zum Erwerb von Handlungsfähigkeit, Selbstbestimmung und Selbstverwirklichung; Gestaltung von Lebensräumen und Sozialbeziehungen zur Förderung von Selbstbildungsprozessen (12), von Integration und zur Überwindung von Krisen (13).

Ziel von Partizipation ist die verbesserte Lebensqualität. Diese Lebensqualität im Alter ist auch von Ausmaß und Art individueller Teilhabe an der Mitgestaltung gesellschaftlicher Räume und der Bewältigung individueller Lebenslagen bestimmt. (14)

Die partizipative Forschung ist gekennzeichnet von einem kollaborativen, interdisziplinären Prozess, mit dem Ziel gemeinsamer Erkenntnisse von Forschenden und Mitforschenden sowie eines produktiven Austauschverhältnisses von Theorie- und Praxiswissen. Im Zentrum steht die Konstituierung eines kommunikativen, öffentlichen Raumes, in der gemeinsam gelebte Alltags- und Arbeitspraxis erforscht wird. Für die methodischen Entscheidungen sind neben Erkenntnisinteresse und Fragestellung die Ressourcen und Möglichkeiten der Adressaten - also der Mitforschenden - in den Blick zu nehmen. (15)

Das Prinzip des Multi-voicing (16) besagt, dass alle Beteiligten ihre Meinung frei äußern sowie gleichberechtigt teilnehmen und mitentscheiden können. Voraussetzung einer Auseinandersetzung auf Augenhöhe ist die Vermittlung analytischer Kompetenzen und theoretischer Konzepte.

\section{So kann es gehen - eine Methode zur partizipativen Forschung}

Leitgedanke einer partizipativen Forschung ist, dass ältere Menschen bereit und fähig sind, sich in ihrem Stadtteil zu engagieren, sobald damit Erwartungen an eine höhere Selbstbestimmung und verbesserte Lebensqualität verbunden wird.

Aus einer Vielzahl sozialräumlicher Methoden bieten sich insbesondere vier für die Arbeit mit Älteren an: die Nadelmethode, die Stadtteilbegehung, die strukturierte Stadtteilbegehung als auch Interviews in Institutionen und mit Schlüsselpersonen. (17)

Exemplarisch soll die Methode der Stadtteilbegehung mit älteren Menschen dargestellt werden, welche der lebensweltorientierten Jugendhilfe nach Ortmann entlehnt ist. Bei der Durchführung begehen Forscher und eine kleine Gruppe Mitforschender gemeinsam den Stadtteil und dokumentieren währenddessen ihre Wahrnehmungen und Interpretationen über die Qualitäten der Sozialräume. (18)

Die Zielrichtung der Stadtteilbegehung und die gewünschte Erkenntnisgewinnung beziehen sich auf lebensweltliche Bewertungen, Deutungen und Interpretationen von Räumen, der Infrastruktur oder vorhandener Institutionen und Angebote durch die Mitforschenden. (19) Nach Böhnisch muss hierbei bedacht werden, dass oftmals die »räumliche Umwelt schon besetzt, gesellschaftlich vordefiniert und funktionalisiert « (20) vorgefunden wird. Diese Vorbestimmung kann sich negativ auf die Nutzung von Räumen und auf das Mobilitätsvermögen älterer Menschen auswirken, ist somit möglicherweise einschränkend statt Handlungschancen erweiternd.

Eine gemeinsame Vorbereitungsphase mit den Adressaten ist zur Realisierung der Partizipation erforderlich, so auch Krisch. Die Zielrichtungen der Begehungen müssen ausgehandelt und klar sein. Dementsprechend gilt es die

\section{Eine weitere Methode zur Sozialraum-Erforschung: die Nadelmethode}

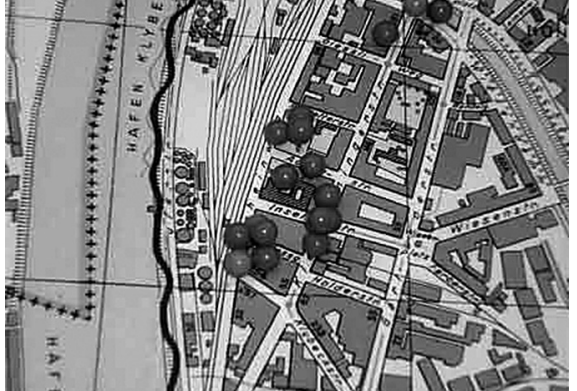

"Die Nadelmethode ist ein Verfahren zur Visualisierung von bestimmten Orten und Plätzen (wie z. B. Wohngegenden oder Treff- und Streifräumen), die jederzeit in der Jugendarbeit angewandt werden kann und augenblicklich zu Ergebnissen führt. Bei dieser aktivierenden Methode, die von Norbert Ortmann entworfen wurde, werden von Kindern, Jugendlichen oder anderen Zielgruppen auf Karten von Stadtteilen/Sozialräumen bestimmte Orte mit Hilfe farbiger Stecknadeln markiert. Werden entsprechend bestimmter Kriterien, wie Alter oder Geschlecht, Nadeln in unterschiedlichen Farben verwendet, sind nach Abschluss des Projektes differenzierte Aussagen - beispielsweise über von Mädchen präferierten oder von jüngeren Jugendlichen gemiedenen Orte - möglich.

Quelle: Ulrich Deinet, Richard Krisch: Nadelmethode. www.sozialraum.de/nadelmethode.php 


\section{Literatur}

Bergold, Jarg/Thomas, Stefan (2010): Partizipative Forschung. In: Mey, Günter/Mruck, Katja (Hg.): Handbuch Qualitative Forschung in der Psychologie, Wiesbaden: VS Verlag, Seite 333-344.

Bertelsmann Stiftung (2008) (Hg.): Alter neu denken. Gesellschaftliches Altern als Chance begreifen, 2. Auflage, Gütersloh: Verlag Bertelsmann Stiftung.

Bundesministerium für Familie, Senioren, Frauen und Jugend (Hg.) (2010): Eine neue Kultur des Alterns. Altersbilder in der Gesellschaft, Erkenntnisse und Empfehlungen des Sechsten Altenberichts.

\section{Der Bundesminister für Jugend, Familie,} Frauen und Gesundheit (Hg.) (1990):

8. Jugendbericht. Bericht über Bestrebungen und Leistungen der Jugendhilfe.

Gintzel, Ullrich (2008): Partizipation. In: Kreft, Dieter/Mielenz, Ingrid (Hg.): Wörterbuch Soziale Arbeit. Aufgaben, Praxisfelder, Begriffe und Methoden der Sozialarbeit und Sozialpädagogik, 6. überarbeitete und aktualisierte Auflage, Weinheim und München: Juventa Verlag, Seite 635-639.

Knopp, Reinhold/Deinet, Ulrich (2006): Leben im Alter. Lösungen für das Quartier. In: Beltz, Julius (Hg.): Sozialmagazin. Die Zeitschrift für Soziale Arbeit, Sozialraum, Altenarbeit mit neuen Mitteln, 11/2006, Juventa Verlag: Weinheim, Seite 14-22.

Knopp, Reinhold (2009): Sozialraumerkundung mit Älteren. In: Deinet, Ulrich (Hg.): Methodenbuch Sozialraum, Wiesbaden: VS Verlag für Sozialwissenschaften, Seite155-164.

\section{Köster, Dietmar/Rüßler, Harald/Stiel, Janina}

(2012): Lebensqualität und Partizipation Älterer im Wohnquartier. In: Otto, Hans-Uwe/Thiersch, Hans (Hg.): neue praxis. Zeitschrift für Sozialarbeit, Sozialpädagogik und Sozialpolitik, 4/12, Lahnstein: Verlag neue praxis, Seite 409-425. Krisch, Richard (2002): Methoden einer sozialräumlichen Lebensweltanalyse. In: Deinet, UIrich/Krisch, Richard (Hg.): Der sozialräumliche Blick der Jugendarbeit. Methode und Bausteine zur Konzeptentwicklung und Qualifizierung, Opladen: Leske und Budrich, Seite 87-154.

Naegele, Gerhard (2011): Alter. In: Otto, HansUwe/Thiersch, Hans (Hrsg.): Handbuch Soziale Arbeit, Seite 32-47.

Schnurr, Stefan (2011): Partizipation. In: Otto, Hans-Uwe/Thiersch, Hans (Hg.): Handbuch Soziale Arbeit, Seite 1069-1078.

Statistisches Bundesamt (2012): Sterbefälle. https://www.destatis.de/DE/ZahlenFakten/ GesellschaftStaat/Bevoelkerung/Sterbefaelle/ Tabellen/Lebenserwartung.pdf?

blob=publicationfile (17.02.2013).
Route zu planen, einen zeitlichen Rahmen zu setzen, der zwei bis drei Stunden nicht überschreiten sollte, sowie die Größe und Zusammensetzung der Gruppen festzulegen. (21)

Da die Nutzungsformen der Räume eines Stadtteils und insbesondere die körperliche Konstitution älterer Menschen sehr unterschiedlich sind, werden Begehungen mit verschiedenen Gruppen, nach der Einteilung der Älteren von Knopp und Deinet, empfohlen.

Mit dem konsequenten Ziel der Befähigung ist es anzuregen, dass die Mitforschenden selbst die Erkenntnisse bezüglich ihrer Aneignungs- und Nutzungsformen festhalten. Dokumentiert werden kann mit Fotoapparaten (22), Videokameras, Aufnahmegeräten sowie Zettel und Stift.

Nach mehreren Stadtteilbegehungen können vielfältige Perspektiven und Interpretationen zu einem verdichteten Bild über die sozialräumlichen Qualitäten entwickelt und schließlich öffentlich präsentiert werden. Dadurch kann für die welt und ihres Sozialraums und werden als solche wertgeschätzt. Ihre subjektiven Meinungen, Bewertungen und Ideen sind von zentraler Bedeutung für das Gelingen der Methode und für einen nachhaltigen Effekt.

- Mitwirkung und Planung: Die Einteilung der Älteren in Gruppen nach Knopp und Deinet bieten für den gemeinsamen Arbeitsprozess unterschiedliche Mitwirkungsmöglichkeiten. Für die ersten beiden Gruppen jüngerer Älterer besteht die Chance sich bei konkreten Planungsvorhaben und perspektivischen Umgestaltungen aktiv einzubringen. Diese Gruppe setzt sich gleichermaßen für ihre eigenen Interessen und die Belange der älteren bis hochbetagten Mitmenschen des Stadtteils ein. Sie agiert somit stellvertretend. Die Gruppen, der in ihrer Mobilität eingeschränkten und auf Hilfe angewiesenen Menschen, werden als Experten für die Fragen nach Barrierefreiheit, sicheren Anlaufstellen

\section{"Ziel von Partizipation ist eine verbesserte Lebensqualität»}

Belange und Bedarfe älterer Menschen sensibilisiert und ein größeres Verständnis bei anderen Bewohnern und Generationen eines Stadtteils erzeugt werden. Forderungen nach veränderter Infrastruktur und modifizierten Angeboten lassen sich damit ebenfalls verbinden. (23)

\section{Das Ziel: Handlungsspielräume eröffnen}

Aus der Durchführung der Stadtteilbegehung und der Fokussierung der Zielgruppe älterer Menschen in ihrem Sozialraum resultiert eine Vielzahl an Handlungsspielräumen. Nicht nur die Adressaten können teilhaben und mitbestimmen, sondern auch alle weiteren Beteiligten, Institutionen und Fachkräfte.

- Aktivierung der Adressaten: Zunächst werden die Mitforschenden selbst zum Handeln aufgerufen und ermächtigt, in dem sie sich an der Vorbereitung, Durchführung, Auswertung und Ergebnispräsentation einer Stadtteilbegehung beteiligen. Sie sind die Experten ihrer Lebens- sowie Ruhe- und Erholungspunkten im Stadtteil und der Größe ihres nutzbaren Nahraums verstanden.

- Kommunikation stärken: Menschen eines Sozialraums kommen über die Begehung miteinander ins Gespräch und werden in der Kommunikation gestärkt. Neue Impulse für das Gemeinschaftsgefühl beeinflussen das Zusammenleben positiv. Kommunikation und Kooperation finden auch zwischen den Mitforschenden, Fachkräften und Institutionen vor Ort statt. In Zusammenarbeit mit Behörden, Stadtplanern, Quartiersmanagement und Sozialer Arbeit können Defizite in der Infrastruktur aufgegriffen und behoben werden. Auf der Basis ermittelter Bedarfe ist es möglich neue Konzepte und Angebote für die Zielgruppen im Sozialraum zu diskutieren und verwirklichen.

- Soziale Netzwerke und Infrastruktur: Partizipatives Handeln in der vorgestellten Form stärkt soziale Netzwerke und fördert Zusammenhalt, gemeinsame Interessen als auch die Identifikation mit dem Stadtteil. 
»Wer die Erfahrung macht, in stadtteilbezogenen Bürgerbeteiligungsverfahren informiert und qualifiziert mitwirken zu können, wird in sich auch das Potenzial erkennen, in anderen Bereichen Mitsprache einzufordern. Wer sich intensiv mit seinem Quartier auseinandergesetzt hat, wird sich dort auch sicherer bewegen und ist offener für Nachbarschaftskontakte", so Knopp. (24)

Resümee: Mit partizipativen Ansätzen im Sozialraum - wie der beteiligenden Stadtteilbegehung - lassen sich unter Einbeziehung älterer Menschen und ihrer Kompetenzen sowohl die Lebensqualität für die einzelnen Bewohner als auch für das gesamte Sozialwesen verbessern.

\section{Anmerkungen}

(1) Bertelsmann Stiftung, 2008, 298.

(2) Vgl. Bundesministerium für Familie, Senioren, Frauen und Jugend, 2010, 6.

(3) Vgl. Knopp u. a., 2006, 16.

(4) Vgl. Naegele, 2011, 34 und Vgl. Statistisches Bundesamt, 2012, 8.

(5) Vgl. Naegele, 2011, 35.

(6) Vgl. Naegele, 2011, 35.

(7) Vgl. Gintzel, 2008, 635.

(8) Vgl. Schnurr, 2011, 1069.

(9) Vgl. Gintzel, 2008, 635.

(10) Vgl. Schnurr, 2011, 1071.

(11) Vgl. beispielsweise Schnurr, 2011, 1069 und 1072 und Vgl. Der Bundesminister für Jugend, Familie, Frauen und Gesundheit, 1990, 88.

(12) Vgl. Schnurr, 2011, 1072.

(13) Vgl. Gintzel, 2008, 635.

(14) Klöster u.a., 2012, 411.

(15) Vgl. Bergold u.a., 2010, 333 ff.

(16) Vgl. Bergold u.a., 2010, 338.

(17) Nähere Ausführungen hierzu in: Krisch, 2002, 87 ff.

(18) Vgl. Knopp u. a., 2006, 18.

(19) Vgl. Krisch, 2002, 87.

(20) Zitiert nach Böhnisch in Krisch, 2002, 88.

(21) Vgl. Krisch, 2002, 93.

(22) Nähere Ausführungen hierzu in: Krisch, 2002, 137 ff.

(23) Vgl. Krisch, 2002, 96 ff.

(24) Knopp, 2009, 163.

\section{Termine}

Bundeskongress Allgemeiner Sozialer Dienst 2013. 4. bis 6. September 2013 in München. Deutscher Verein für öffentliche und private Fürsorge e. V. www.deutscher-verein.de

Gesprächsführung in Konfliktsituationen in Arbeitsbeziehungen, Gruppen und Teams. 4. bis 6. September 2013 in Mainz. Katholische Fachhochschule Mainz. www.kfh-mainz.de

"Zwischen Gewinnen und Verlieren“ Systemische Kernkompetenzen bei unvereinbaren Erwartungen und Zwangskontexten. 9. bis 11. September 2013 in Eisenach. Evangelischer Erziehungsverband (EREV). www.erev.de

Aktuelle fachliche, fachpolitische und rechtliche Entwicklungen in der Sozialhilfe. 16. bis 18. September 2013 in Berlin. Deutscher Verein für öffentliche und private Fürsorge e. V. www.deutscher-verein.de

Praktisches Beschwerdemanagement. 18. und 19. September 2013 in Engelskirchen (Nordrhein-Westfalen). www. caritas-akademie.de

Studienreise: Sozialarbeit in New York City. 21. bis 29. September 2013. www.akademie.org

Empowerment - mit frischem Wind neue Fahrt aufnehmen! 25. bis 27. September 2013 in Berlin. AWO-Bundesakademie. www.akademie.awo.org

Sozialraumorientierung in Sozialen Diensten der Jugendhilfe. 25. bis 27. September 2013 in Berlin. LüttringHaus, Institut für Sozialraumorientierung, Quartier- und Case Management (DGCC) Essen. www.luettringhaus.info

Führen und Leiten für Einsteiger. Rollen und Aufgaben der Führungskraft, Mitarbeitergespräche als Führungsinstrument, Grundhaltungen und Menschenbild, praktische Übungen und Reflexion der eigenen beruflichen Praxis. 26. September 2013 in Stuttgart. www.wohlfahrtswerk.de

Systemisches Denken und Handeln im Allgemeinen Sozialen Dienst. 7. und 8. Oktober 2013 in Münster. www.fh-muenster.de
Ressourcen und Resilienz bei Menschen in Armut und Wohnungsnot fördern. 9. bis 11. Oktober 2013 in Berlin. Führungsakademie für Kirche und Diakonie gemeinnützige AG. www.fakd-dm.de

Typische Probleme bei der Führung und Abwicklung einer rechtlichen Betreuung. Seminar für Fachkräfte bei Betreuungsbehörden und Betreuungsvereinen, Berufsbetreuerinnen und Berufsbetreuer. 21. Oktober 2013 in Herrenberg-Gültstein (Baden-Württemberg). www.kvjs.de

Gekonnt kontern. Selbstsicher Gespräche führen. 28. und 29. Oktober 2013 in Berlin. Kommunales Bildungswerk e. V. www.kbw.de

Krisenfest und rentabel: Investition in Bildung Persönlichkeitsbildung lohnt sich wieder, Seminar für Fachkräfte der pädagogischen und Sozialen Arbeit. 30. und 31. Oktober 2013 in Berlin. www.akademie.org

Einführung in systemisches Arbeiten mit Eltern, Jugendlichen und Kindern in der Jugendhilfe. 4 . bis 6 . November 2013 am Timmendorfer Strand. www.erev.de

Vernetzte Lebenswelten. 12. Praxistage der Kinder- und Jugendlichenpsychotherapie der Deutsche Gesellschaft für Verhaltenstherapie e. V. 9. und 10. November 2013 in Stuttgart. www. dgvt-fortbildung.de

Erfolgreiche Pressearbeit. 12. November 2013 in München. Institut für Beratung und Projektentwicklung e. V. www.ibpro.de

Verändern durch Verstehen - klientenzentrierte Gesprächsführung. 21. und 22. November 2013 in Berlin. Paritätische Akademie. www.akademie.org

Kennzahlensysteme und Controlling im sozialen Bereich. 28. und 29. November 2013 in Frankfurt am Main. Fort- und Weiterbildungsinstitut der Arbeiterwohlfahrt Kreisverband Frankfurt am Main e. V. www.awofrankfurt.de/awo/Bildung/FWIA 